\title{
Travelling Salesperson Problem for dynamic systems
}

\author{
Sleiman Itani ${ }^{*}$ Emilio Frazzoli $*$ Munther A Dahleh* \\ * LIDS, Massachusetts Institute of Technology, Cambridge, 02139 USA \\ (ssolomon@mit.edu).
}

\begin{abstract}
:
In this paper, we study the following version of the Travelling Salesperson Problem: Find the fastest closed trajectory for a controlled dynamic system such that its output visits all points in a given (finite) set. We present an algorithm that, if the $n$ points are randomly sampled from a uniform distribution, produces an output trajectory the expected duration of which scales within a constant factor of the optimum asymptotically in $n$.
\end{abstract}

\section{INTRODUCTION}

The problem we are tackling in this paper has two components; the first is the dynamic system and the second is the Travelling Salesperson Problem (TSP). Problems similar to this, that inject the kinematics or dynamics into the study of the TSP or similar problems, are gaining more attention in the literature. Historically, the TSP has generally been studied as a combinatorial optimization problem, and its objective has been to minimize the length of a tour through a given set of points. Recently though, the properties the system that has to tour the points have been considered; and the TSP has been recently studied for the Dubins vehicle [1, 10], the double integrator [2], the Reeds-Shepp car, and the differential drive robot [7].

With all the advances in robotics and the growth of interest in Unmanned Aerial Vehicles (UAV's), the applications that need a fusion of dynamics and optimal path planning through a set of points are countless. The possible use of robots and UAV's in search and rescue missions, surveillance and many other applications that require optimized planning of a route make the problem we are tackling important for the near future. In addition to that, studying the TSP for dynamic systems might also offer insight to the solution of different path planning problems for dynamic systems. Lastly, injecting the dynamics into the TSP is a natural step in the evolution of the research on the TSP and similar problems, where the constraints on the system that will tour the points are added to the optimal path planning problem that was historically solved without those constraints.

In this paper, we model the dynamic systems with state space models that are affine in control. The class of systems that are affine in control is very general, and can be used to model a wide range of vehicles, robots and other machines. This makes this class of systems an interesting and natural family of models to introduce into the framework of the TSP. Systems that are affine in control have been widely studied in the literature, due to their elegance, simplicity and wide scope. Much of the research on such systems targets their reachability and steering properties; those aspects of dynamical systems are very interesting for problems that seek an optimal path through a set of points.

Our previous work [10] and the work of K. Savla et al. [1] studied similar problems for the Dubins vehicle. It was proved that the expected length of the optimal TSP tour for the Dubins vehicle is $\Theta\left(n^{2 / 3}\right)^{1}$, where $n$ is the number of points the vehicle is required to visit. That result was reached by incorporating the structure of the motion of the Dubins vehicle $[3,4,6]$ into the TSP problem and using that structure in the design of the algorithm. We also solved other vehicle routing problems for the Dubins vehicle, like the Dynamic Repairman Problem. Our work here is in some sense a generalization of the first part of that work to a much richer class of systems.

The rest of this paper is organized as follows: In Section 2 we introduce the notation, prepare the ground for our study by citing some relevant results, and define the problem rigorously. Section 3 studies the area of the smalltime reachable set and local point-to-point steering of the dynamic system. The understanding of those sub-problems is essential for the problem at hand. Section 4.1 provides the lower bound on the expected TSP time for a system that is affine in control, and in Section 4.2 introduces an algorithm that produces a tour whose time is within a constant factor of the bound in Section 4.1. Section 5 has the conclusions and the future work.

\section{NOTATION AND PROBLEM STATEMENT}

In this paper, we model the dynamic systems that we are studying with state space models that are affine in control and have an output in $\mathbb{R}^{2}$. Thus they are described as follows:

$$
\begin{gathered}
\dot{x}=g_{0}(x)+\sum_{i=1}^{m} g_{i}(x) u_{i}, \\
y=h(x),
\end{gathered}
$$

1 We say a function $f(n)$ is $O(g(n))$ if $\exists c, N>0$ such that $f(n) \leq$ $c g(n) \forall n>N$, we say $f(n)$ is $\Omega(g(n))$ if $g(n)$ is $O(f(n))$ and we say $f(n)$ is $\Theta(g(n)) f(n)$ is $O(g(n))$ and $\Omega(g(n))$. We say $f(l)$ is $o(g(l))$ if $\lim _{l \rightarrow 0} \frac{f(l)}{g(l)}=0$ (for functions) or $\lim _{n \rightarrow \infty} \frac{f(n)}{g(n)}=0$ (for sequences) 


$$
\begin{gathered}
x(0)=x_{0}, \\
x \in \mathbb{R}^{p}, y \in \mathbb{R}^{2}, u_{i} \in \mathbb{U}, \\
\mathbb{U}=\{u(.): \mathbb{R} \rightarrow[-M, M]\} .
\end{gathered}
$$

We are interested in studying the TSP tour of such systems, and how the dynamics of the system affect the expected value of the optimal time duration. These statements will be made more rigorous in the following section.

\subsection{Problem Statement}

Given a dynamic system that is modelled as (1), a closed, bounded region $R$ (assumed to be rectangular with height $H$ and width $W$, without loss of generality) in the output space of the system, and a set of points $P=\left\{p_{i} \in\right.$ $R, i=1, \ldots, n\}$. We study $\mathcal{C}_{P}$, the fastest output curve of system (1) that passes through all of the points in $P$. More specifically, we are interested in the expected time it takes the system to trace $\mathcal{C}_{P}$ if the $p_{i}$ 's are randomly and uniformly distributed over $R$. We also seek an algorithm that produces an output curve the expected time duration of which scales in terms of $n$ like the optimum does (for large $n$ ).

Next, we introduce some terminology and definitions for systems that are affine in control; most of these definitions are classical in the literature [8] [9]. We start by introducing the most basic object we need, the reachable set of a dynamic system.

\section{Definition 1. Reachable set:}

Given $T \geq 0$, the reachable set from state $x_{0}$ for a dynamic system is the set $R_{T}\left(x_{0}\right)$ of states $x$ such that $\forall x_{1} \in R_{T}$, $\exists u_{1}^{*}, u_{2}^{*}, \ldots, u_{m}^{*} \in \mathbb{U}$ such that:

$$
\begin{gathered}
x(0)=x_{0}, x(T)=x_{1}, \\
x(t) \in R_{T} \forall t<T .
\end{gathered}
$$

This is the set of states that are reachable in exactly $T$. We define the set of states reachable in time less than or equal to $T$ by:

$$
R_{\leq T}\left(x_{0}\right)=\cup_{0 \leq t \leq T} R_{T}\left(x_{0}\right) .
$$

We extend the previous definition to the output space, and so we define the output-reachable set from a state $x_{0}$ to be the set $O_{T}\left(x_{0}\right)$ of points

$$
y=h(x), x \in R_{T}\left(x_{0}\right),
$$

and

$$
O_{\leq T}\left(x_{0}\right)=\cup_{0 \leq t \leq T} O_{T}\left(x_{0}\right) .
$$

We turn to some important properties of some systems that are affine in control.

\section{Definition 2. Small-time Locally Controllable Systems:}

A system is small-time locally controllable at $x_{0} \in \mathbb{R}^{p}$ if $\exists T>0$ such that

$$
x_{0} \in \operatorname{Interior}\left(R_{\leq t}\left(x_{0}\right)\right) \forall t \text { such that } 0<t \leq T .
$$

We call a system small-time locally controllable if it is small-time locally controllable at all $x \in \mathbb{R}^{p}$.
We also extend the previous definition to the output space, and say that a system is output small-time locally controllable at $x_{0}$ if $\exists T>0$ such that

$$
h\left(x_{0}\right) \in \operatorname{Interior}\left(O_{\leq t}\left(x_{0}\right)\right) \forall t \text { such that } 0<t \leq T \text {. }
$$

Definition 3. Vector Fields:

For all the purposes of this work, a vector field $f(x)$ is an infinitely differentiable mapping from $\mathbb{R}^{p}$ to $\mathbb{R}^{p}$.

Given a vector field $f$ and a function $w: \mathbb{R}^{p} \rightarrow \mathbb{R}$, we denote the derivative of $w$ along $f$ by :

$$
\mathcal{L}_{f} w(x)=\sum_{i=1}^{p} \frac{\partial w(x)}{\partial x_{i}} f_{i}(x) .
$$

Given a vector field $f$ and $g: \mathbb{R}^{p} \rightarrow \mathbb{R}^{q}$, we call the derivative of $g$ along $f$ the new $\mathbb{R}^{p} \rightarrow \mathbb{R}^{q}$ function:

$$
\mathcal{L}_{f} g(x)=\frac{\partial g}{\partial x} f(x)
$$

Note that the $i^{\text {th }}$ component of $\mathcal{L}_{f} g$ is the derivative of the function $g_{i}$ along $f$. Thus the use of similar notation should not be confusing.

A simple piece of notation that we will use is the function $x_{j}(x)$ which extracts the $j^{t h}$ component of $x$. Notice that

$$
\mathcal{L}_{f} x_{j}(x)=f_{j}(x) .
$$

Also, we use $s_{f, T}\left(x^{0}\right)$ to denote the integral curve of $f$ passing through $x^{0}$ (if it exists) at time $T$, so $s_{f, T}\left(x^{0}\right)$ is the solution of the differential equation

$$
\frac{d}{d t} x(t)=f[x(t)], \quad x(0)=x^{0}
$$

at the time $T$.

We now solve two sub-problems whose solution is essential to our study. One is helpful in finding a lower bound on the expected time the system needs to trace $\mathcal{C}_{P}$, and the other is an important piece of our algorithm to produce an output curve whose time duration is close to the lower bound.

\section{SMALL-TIME REACHABILITY AND STEERING}

This section is divided into two parts: In the first part, we bound how the area of the output-reachable set $O_{\leq T}$ (definition 1) of system (1) scales in terms of $T$ as $T \rightarrow 0$. In the second part, we study how to steer system (1) between two points that are close together.

\subsection{Small-Time Reachability}

In order to study the area of the output-reachable set $\left(A_{\leq T}(y)\right)$, we will use a change of coordinates in the output plane to achieve some simplifying properties.

Let $o_{1}$ and $o_{2}$ be the two smallest natural numbers that satisfy:

$$
\begin{aligned}
& f_{1}=\mathcal{L}_{g_{i_{0}}} \ldots \mathcal{L}_{g_{i_{o_{1}-1}}} h\left(x_{0}\right) \neq 0, \\
& f_{2}=\mathcal{L}_{g_{j_{0}}} \ldots \mathcal{L}_{g_{j_{o_{2}-1}}} h\left(x_{0}\right) \neq 0,
\end{aligned}
$$


and $f_{1}$ and $f_{2}$ are linearly independent, where

$$
i_{0}, \ldots, i_{o_{1}-1}, j_{0}, \ldots, j_{o_{2}-1} \in\{0, \ldots, m\} .
$$

We assume $o_{1} \leq o_{2}$ without loss of generality, and define the new coordinates $y_{1}^{*}$ and $y_{2}^{*}$ as follows:

(1) $y_{2}^{*}$ is the part of $f_{2}$ that is orthogonal to $f_{1}$ (i.e., $\left.f_{2}-\frac{f_{2} \cdot f_{1}}{\left|f_{2}\right|^{2}} f_{1}\right)$.

(2) $y_{1}^{*}$ is any unit vector in the output space that is orthogonal to $y_{2}^{*}$.

After the change of coordinates, $y_{1}^{*}$ and $y_{2}^{*}$ satisfy the following properties for $s \in\{1,2\}$ :

(1)

$\forall k$ such that $0 \leq l \leq k<o_{s}-1, i_{l} \in\{0,1,2, \ldots, m\}$,

$$
\mathcal{L}_{g_{i_{0}}} \ldots \mathcal{L}_{g_{i_{k}}} h_{s}^{*}\left(x_{0}\right)=0 \text {. }
$$

(2) $\exists i_{0}, i_{1}, \ldots, i_{o_{s}-1}$ such that:

$$
\mathcal{L}_{g_{i_{0}}} \ldots \mathcal{L}_{g_{i_{o_{s}-1}}} h_{s}^{*}\left(x_{0}\right) \neq 0 \text {. }
$$

Here, $h$ has been changed to $h^{*}$ because of the change of coordinates $\left(y^{*}=h^{*}(x)\right.$ replaces $\left.y=h(x)\right)$. From this point on, we will assume that $o_{1}, o_{2}, y_{1}^{*}$ and $y_{2}^{*}$ are invariant over $x \in \mathbb{R}^{p}$, and that $g_{i}, i \in\{0, \ldots, m\}$ and all of its derivatives (with respect to $x$ ) are bounded. We will drop the ${ }^{*}$ from this point forward to simplify the notation, assuming that this change of coordinates has been applied. Note that the change of coordinates does not affect how the area of the small-time output-reachable set $O_{\leq T}$ scales in terms of $T$. It only changes it by a factor that is independent of $T$.

Theorem 1. Given a system described in (1) and $o_{1}$ and $o_{2}$ as above, $\exists C_{U}, C_{L}>0$ such that:

$$
C_{L} \leq \lim _{T \rightarrow 0} \frac{A_{\leq T}}{T^{o_{1}+o_{2}}} \leq C_{U}
$$

To prove theorem 1, we will first prove that for $s \in\{1,2\}$, there exists $C_{U_{s}}$ such that the distance $d_{s}(t)$ that the output of system (1) can move in direction $x_{s}$ in time $t$ satisfies the following lemmas:

Lemma 1. $\exists T>0, C_{U_{s}}>0$ such that $\forall t \leq T$

$$
\frac{d_{s}(t)}{t^{o_{s}}} \leq C_{U_{s}} \text {. }
$$

and then prove that $\exists C_{L_{s}}$ such that $d_{s}$ satisfies:

Lemma 2. $\exists T>0, C_{U_{s}}>0$ such that $\forall t \leq T$

$$
\frac{d_{s}(t)}{t^{o_{s}}} \geq C_{L_{s}} \text {. }
$$

The upper bound on the area of the reachable set is important for the lower bound on the expected time the system needs to trace $\mathcal{C}_{P}$. The lower bound is useful for the steering algorithm we will use as a sub-algorithm in Section 4.2.1.

The following two subsections will have the proofs of statements (1) and (2). We will start by quoting an interesting result, which is central to our proof [8]:

Theorem 2. The output of system (1) evolves as follows:

$$
y_{j}(t)=h_{j}\left(x_{0}\right)
$$

$$
+\sum_{k=0}^{\infty} \sum_{i_{0}, \ldots, i_{k}=0}^{m} \mathcal{L}_{g_{i_{0}}} \ldots \mathcal{L}_{g_{i_{k}}} h_{j}\left(x_{0}\right) \int_{0}^{t} d \xi_{i_{k}} \ldots d \xi_{i_{0}}
$$

Where the integral in (3) is defined iteratively by:

$$
\begin{gathered}
\xi_{0}(t)=t \\
\xi_{j}(t)=\int_{0}^{t} u_{j}(\tau) d \tau \\
\int_{0}^{t} d \xi_{i_{k}} \ldots d \xi_{i_{0}}=\int_{0}^{t} d \xi_{i_{k}}(\tau) \int_{0}^{\tau} d \xi_{i_{k-1}} \ldots d \xi_{i_{0}} .
\end{gathered}
$$

Upper Bound on the Area of the Reachable Set

Lemma 3. Given system (1), for $s \in\{1,2\}$ and $o_{s}$ as described above, then $\exists T, C_{U_{s}}>0$ such that $\forall t<T$ and $u(.) \in \mathbb{U}$,

$$
\left|y_{s}(t)-y_{s}(0)\right|<C_{U_{s}} t^{o_{s}}
$$

Proof 1. From the definition of $o_{s}$, we have that $\forall k$ such that $0 \leq k<o_{s}, i_{l} \in\{0,1,2, \ldots, m\}$,

$$
\mathcal{L}_{g_{i_{0}}} \ldots \mathcal{L}_{g_{i_{k}}} h_{s}\left(x_{0}\right)=0 .
$$

Inserting this result in (3) produces:

$$
\begin{gathered}
y_{s}(t)=y_{s}(0) \\
+\sum_{k=o_{s}-1}^{\infty} \sum_{i_{0}, \ldots, i_{k}=0}^{m} \mathcal{L}_{g_{i_{0}}} \ldots \mathcal{L}_{g_{i_{k}}} h_{s}\left(x_{0}\right) \int_{0}^{t} d \xi_{i_{k}} \ldots d \xi_{i_{0}} .
\end{gathered}
$$

To establish the bound, we use the following two facts:

(1) Since $g_{i}(),. x_{j}($.$) , and their partial derivatives of any$ order with respect to $x$ are bounded around $x_{0}$, $\exists M_{1}, M_{2}$ such that:

$$
\mathcal{L}_{g_{i_{0}}} \ldots \mathcal{L}_{g_{i_{k}}} x_{j}\left(x_{0}\right) \leq M_{1} M_{2}^{k} .
$$

(2) Since $\left|u_{j}().\right| \leq M$,

$$
\int_{0}^{t} d \xi_{i_{k}} \ldots d \xi_{i_{0}} \leq \frac{(M t)^{k+1}}{(k+1) !}
$$

Therefore, $\exists T$ small enough such that the sum is convergent.

Using in the bounds from (4) and (5), we get:

$$
x_{j}(x(t))-x_{j}\left(x_{0}\right) \leq \sum_{k=o_{s}-1}^{\infty} \frac{m M_{1}}{M_{2}(k+1) !}\left(M M_{2} t\right)^{k+1}
$$

and the result directly follows.

The two dimensional version follows directly. Therefore, given an output $y_{0}$, we define the order of the area at $y_{0}$, $o\left(y_{0}\right)$ as:

$$
o\left(y_{0}\right)=\sup _{\left\{x \in \mathbb{R}^{p}: y_{0}=h(x)\right\}} o_{1}(x)+o_{2}(x) .
$$

We now turn to the lower bound on the area of the smalltime output-reachable set. The bounds on the individual motions (in the directions of $y_{1}$ and $y_{2}$ ) are actually what we are interested in. 


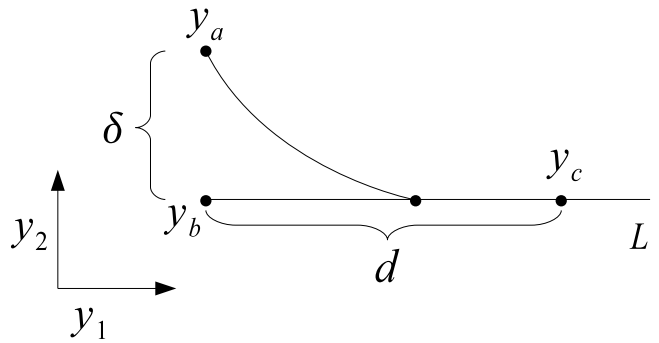

Fig. 1. Steering the output locally

3.2 Lower bound on the area of the reachable set and steering

In this section, we study the implications of statement (2) on the small-time maneuverability of system (1). We will leave the proof of statement (2) to the appendix.

Consider the situation in Figure 1, where the system's output is steered from $y_{a}$ to $y_{c}$, and we have $\delta=\frac{\left|\left(y_{a}-y_{c}\right) \cdot y_{2}\right|}{\left|y_{2}\right|}$ and $d=\frac{\left|\left(y_{a}-y_{c}\right) \cdot y_{1}\right|}{\left|y_{1}\right|}$. Without loss of generality, we assume that if there is a drift, it is in the direction of $y_{1}$ (note that $o_{s}, s \in\{1,2\}$ corresponding to the $f_{s}$ caused by drift is 1 , and so our assumption here doesn't conflict with our previous assumption that $o_{1} \leq o_{2}$ ). The statement that we want to prove is the following:

Proposition 1. If $\delta \leq C_{L_{2}} C_{U_{1}}^{-\frac{o_{2}}{o_{1}}} d^{\frac{o_{2}}{o_{1}}}$, then $\exists C_{m}>0$ such that the output of the system can be steered from $y_{a}$ to $y_{c}$ in time $t=C_{m} d^{\frac{1}{o_{1}}}$.

Proof 2. It is obvious from (2) that the time needed for the output to reach $L$ from $y_{a}$ is at most

$$
t_{1}=\left(\frac{\delta}{C_{L_{2}}}\right)^{\frac{1}{o_{2}}} \text {. }
$$

The system's output could have moved in the $y_{1}$ direction (because of drift or as a side effect of the inputs we used to move in the $y_{2}$ direction) for a distance

$$
d^{*} \leq C_{U_{1}} t^{o_{1}}=C_{U_{1}} C_{L_{2}}^{-\frac{o_{1}}{o_{2}}} \delta^{\frac{o_{1}}{o_{2}}}=d .
$$

Therefore once the system's output is at $L$, it can move along the $y_{1}$ direction to get to $y_{c}$ (since that motion is in the direction of the drift, if there is any). This will take time $t_{2} \leq C_{L_{1}}^{-\frac{1}{o_{1}}} d^{\frac{1}{o_{1}}}$

Thus the output can be steered from $y_{a}$ to $y_{c}$ in time

$$
\begin{gathered}
t=t_{1}+t_{2} \leq\left(\frac{\delta}{C_{L_{2}}}\right)^{\frac{1}{o_{2}}}+\left(\frac{d}{C_{L_{1}}}\right)^{\frac{1}{o_{1}}} \\
\leq\left(C_{U_{1}}^{\frac{1}{o_{1}}}+C_{L_{1}}^{-\frac{1}{o_{1}}}\right) d^{\frac{1}{o_{1}}}
\end{gathered}
$$

when $\delta \leq C_{L_{2}} C_{U_{1}}^{-\frac{o_{2}}{o_{1}}} d^{\frac{o_{2}}{o_{1}}}$.

Therefore, to steer the output between two points, we will use motions like the ones in Figure 1. Of course, we will make sure that $\delta$ and $d$ are small and that $\delta \leq$ $C_{L_{2}} C_{U_{1}}^{-\frac{o_{2}}{o_{1}}} d^{\frac{o_{2}}{o_{1}}}$.

\section{MAIN RESULT:}

We are now ready to present our main result:

Theorem 3. The expected time for system (1) to trace $\mathcal{C}_{P}$ when $P$ has $n$ points is $\Theta\left(n^{1-\frac{1}{o_{1}+o_{2}}}\right)$.

The proof is two parts, we will prove a lower bound on the expected tour time duration in this section and then provide an algorithm whose expected time is within a constant factor of that bound in the next.

\subsection{TSP time lower bound:}

Lemma 4. Let the optimal expected tour time for system (1) be $T_{T S P}$. If the points in $P$ are uniformly distributed, then $T_{T S P}$ is $\Omega\left(n^{1-\frac{1}{o_{1}+o_{2}}}\right)$. Where $o_{1}$ and $o_{2}$ are as defined above.

Proof 3. There are three steps in the proof of lemma 4:

Given that the output of system (1) is at a certain point, let the minimum time needed to travel to a point $p_{i} \in P$ be $t^{*}$. To bound $\mathbf{E}\left[t^{*}\right]$, we consider $\mathbf{P}\left[t^{*}>t\right]$. Using the upper bound on the area of the small time reachable set, the area reachable in time $t$ is less than $C_{U} t^{o_{1}+o_{2}}$. Since the $p_{i}$ 's are uniformly distributed in $R$, it follows that

$$
\mathbf{P}\left[t^{*}>t\right] \geq 1-n \frac{C_{U} t^{o_{1}+o_{2}}}{W H},
$$

where $n$ is the cardinality of $P$.

Where $c=n \frac{C_{U}}{W H}$.

$$
\begin{gathered}
\mathbf{E}\left[t^{*}\right] \geq \int_{0}^{\infty} \mathbf{P}\left[t^{*}>\tau\right] d \tau \\
\geq \int_{0}^{\infty} \max \left\{0,1-c \tau^{o_{1}+o_{2}}\right\} d \tau
\end{gathered}
$$

$$
\begin{aligned}
& \mathbf{E}\left[t^{*}\right] \geq \int_{0}^{c^{-\frac{1}{o_{1}+o_{2}}}} 1-c \tau^{o_{1}+o_{2}} d \tau \\
= & c^{-\frac{1}{o_{1}+o_{2}}}-c \frac{1}{1+o_{1}+o_{2}} c^{-\frac{1+o_{1}+o_{2}}{o_{1}+o_{2}}} \\
= & \frac{o_{1}+o_{2}}{1+o_{1}+o_{2}}\left(\frac{C_{U}}{W H}\right)^{-\frac{1}{o_{1}+o_{2}}} n^{-\frac{1}{o_{1}+o_{2}}} .
\end{aligned}
$$

The expected total travel time is at least $n$ times the expected time to reach the closest point. Therefore,

$$
\begin{gathered}
\mathbf{E}\left[T_{T S P}\right] \geq n \frac{o_{1}+o_{2}}{1+o_{1}+o_{2}}\left(\frac{C_{U}}{W H}\right)^{-\frac{1}{o_{1}+o_{2}}} n^{-\frac{1}{o_{1}+o_{2}}} \\
=C_{T S P}^{L} n^{1-\frac{1}{o_{1}+o_{2}}}
\end{gathered}
$$

where

$$
C_{T S P}^{L}=\frac{o_{1}+o_{2}}{1+o_{1}+o_{2}}\left(\frac{C_{U}}{W H}\right)^{-\frac{1}{o_{1}+o_{2}}} .
$$

This result means the following: $o_{1}$ and $o_{2}$ are a measure of the "difficulty" of moving in two directions in the output space. Thus the higher $o_{1}$ and $o_{2}$ are, the smaller the area of the set reachable in time $t$ is, and so the system will have to travel for a longer time between any two points on average (specifically between any point and its closest neighbor in the proof above), and so the expected time to trace $\mathcal{C}_{P}$ will be higher. 


\subsection{TSP time upper bound:}

We now turn to the upper bound of theorem 3, given by the following lemma:

Lemma 5. $T_{T S P}$ is $O\left(n^{1-\frac{1}{o_{1}+o_{2}}}\right)$.

The proof of lemma 5 is constructive, that is, we provide an algorithm that produces an output curve $\mathcal{C}_{L A}$ for system (1) such that the time needed for the system to trace $\mathcal{C}_{L A}$ is $O\left(n^{1-\frac{1}{o_{1}+o_{2}}}\right)$. For simplicity of presentation, we add a few assumptions here; we assume that $y_{1}$ is parallel to $H$ and that $\exists T_{T}>0$ such that between any two points $y_{1}$ and $y_{2}$ in $R$, the system can be steered from $y_{1}$ to $y_{2}$ in time less than $T_{T}$.

Level Algorithm The algorithm we use to construct the upper bound is a generalization of our algorithm for the TSP for the Dubins vehicle [10], mixed with the one in [1]. We assume that $o_{1} \leq o_{2}$, without loss of generality. The simplest version of the Level Algorithm for system (1) is as follows:

(1) Set level counter $i=1$ and the maximum level $i^{*}=\log _{2}\left(n^{\frac{o_{1}+o_{2}-1}{o_{1}+o_{2}}}\right)-\frac{o_{1}+o_{2}-1}{o_{1}+o_{2}}$.

(2) Let $k_{i}=2^{(i-2)\left(o_{1}+o_{2}\right)}, c_{1}=C_{L_{2}}^{\frac{o_{1}}{o_{1}+o_{2}}} C_{U_{1}}^{\frac{o_{2}}{o_{1}+o_{2}}}$ and $c_{2}=c_{1}^{-1}$. Cover $R$ with rectangles whose width $w_{i}=$ $c_{2}\left(\frac{k_{i} W H}{n}\right)^{\frac{o_{2}}{o_{1}+o_{2}}}$ and length $l_{i}=c_{1}\left(\frac{k_{i} W H}{n}\right)^{\frac{o_{1}}{o_{1}+o_{2}}}$. Note that $c_{1}$ and $c_{2}$ were chosen so that the area of any l-rectangle at level $i$ is $\frac{k_{i} W H}{n}$ and $w_{i}=C_{L_{2}} C_{U_{1}}^{-\frac{o_{1}}{o_{2}}} l_{i}^{\frac{o_{1}}{o_{2}}}$. We call these rectangles l-rectangles.

(3) Visit at least one point in every non-empty l-rectangle by doing two passes, each of which is as follows:

(a) Move along the rows bottom up, alternating in direction.

(b) Visit one point in alternating l-rectangles, using motions as in Section 3.2 .

(4) If $i \leq i^{*}$, increment it by 1 and go to 2 .

(5) If $i>i^{*}$, use a greedy algorithm to pick up the points that are left.

Some important points about the Level Algorithm are the following:

(1) The rectangles are aligned such that their lengths are along $H$ and their widths are along $W$.

(2) Each of the l-rectangles of a certain level $i$ is made of $2^{o_{1}+o_{2}}$ l-rectangles of the previous level. Its width is $2^{o_{2}}$ of theirs and its length is $2^{o_{1}}$ of theirs.

(3) The reason we visit a point in alternating l-rectangles is that in that case we can only use motions like in Section 3.2. This will guarantee that the time travelled in each l-rectangles is less than $C_{m} l_{i}^{\frac{1}{o_{1}}}$.

(4) The number of points not visited after the $\left\lfloor i^{*}\right\rfloor$ level is $n_{l}=O\left(n^{\frac{o_{1}+o_{2}-1}{2\left(o_{1}+o_{2}\right)}}\right)$.

We now turn to the proof that the time the system needs to trace $\mathcal{C}_{L A}$ is $O\left(n^{1-\frac{1}{o_{1}+o_{2}}}\right)$.

Note that because of fact (4), the time needed to clear the points left after the $\left\lfloor i^{*}\right\rfloor^{t h}$ level will not affect the order of the time needed to trace $\mathcal{C}_{L A}$ (since it is less than
$T_{T}\left(n_{l}+1\right)=O\left(n^{\frac{o_{1}+o_{2}-1}{2\left(o_{1}+o_{2}\right)}}\right)$. We therefore just need to prove that the time the system needs to trace $\mathcal{C}_{L A}$ over the levels is $O\left(n^{1-\frac{1}{o_{1}+o_{2}}}\right)$.

Time to trace $\mathcal{C}_{L A}$ To bound the time system (1) needs to trace $\mathcal{C}_{L A}$ over the levels, we start by bounding the time needed to trace $\mathcal{C}_{L A}$ in one level. The bound is given by the following lemma:

Lemma 6. The maximum time needed to trace one pass at level $i$ is less than:

$$
C_{m} W H l_{i}^{\frac{1}{o_{1}}-1} w_{i}^{-1}+o\left(l_{i}^{\frac{1}{o_{1}}-1} w_{i}^{-1}\right),
$$

where $C_{m}$ is the constant from Section

Proof 4. First, we will start by finding the time needed to trace one row of l-rectangles. We know that the number of l-rectangles along $H$ are at most $\frac{H}{l_{i}}+1$.

Therefore, since in every l-rectangle the system used curves as in Section 3.2, the time travelled in traversing one row is bounded by

$$
T_{r}=H l_{i}^{\frac{1}{o_{1}}-1}+l_{i}^{\frac{1}{o_{1}}}+o\left(l_{i}^{\frac{1}{o_{1}}}\right) .
$$

To turn from one row to the other, some additional time bounded by $T_{T}$ is needed.

The number of rows is

$$
N_{r} \leq \frac{W}{w_{i}}+1
$$

To go back to the beginning of first row, the system will also need some additional time bounded by $T_{T}$.

Thus the total time to trace one pass over the whole square is bounded by:

$$
N_{r}\left[T_{r}+T_{T}\right]+T_{T}
$$

which is equal to:

$$
=C_{m} W H l_{i}^{\frac{1}{o_{1}}-1} w_{i}^{-1}+o\left(W H l_{i}^{\frac{1}{o_{1}}-1} w_{i}^{-1}\right)
$$

at level $i$.

Therefore, The length of the time travelled by the vehicle at any level is bounded by two times the maximum time travelled in any certain pass over the rows.

Therefore from Lemma 1 and the fact that

$$
w_{i}=c_{2}\left(\frac{k W H}{n}\right)^{\frac{o_{2}}{o_{1}+o_{2}}}
$$

and

$$
\begin{aligned}
& l_{i}=c_{1}\left(\frac{k W H}{n}\right)^{\frac{o_{1}}{o_{1}+o_{2}}} \text {, } \\
& T_{\text {level } i} \leq 2 C_{m}(W H)^{\frac{1}{o_{1}+o_{2}}}\left(\frac{n}{k}\right)^{1-\frac{1}{o_{1}+o_{2}}}+o\left(n^{1-\frac{1}{o_{1}+o_{2}}}\right)
\end{aligned}
$$

The total time system (1) needs to trace $C_{L A}$ over all of the levels can be bounded by:

$$
T_{\mathrm{LA}} \leq 2(W H)^{\frac{1}{o_{1}+o_{2}}} n^{1-\frac{1}{o_{1}+o_{2}}} \sum_{i=1}^{\left\lfloor i^{*}\right\rfloor} 2^{\left(o_{1}+o_{2}-1\right)(2-i)}
$$




$$
\leq \frac{2^{o_{1}+o_{2}}}{1-2^{1-o_{1}-o_{2}}}(W H)^{-\frac{1}{o_{1}+o_{2}}} n^{1-\frac{1}{o_{1}+o_{2}}}
$$

To complete the proof of lemma 5 , we have to prove fact 4. The proof is actually close to the one in [1], and will be skipped here for the lack of space.

\section{CONCLUSIONS AND FUTURE WORK}

In this paper, we studied the TSP for systems that are affine in control. We proved limits on the small-time reachable area, and from those we derived a lower bound on the minimum expected travel time for a system that is affine in control to visit $n$ uniformly distributed points. We also provided an algorithm that produces a tour that system (1) can trace in time that has the same asymptotic order in $n$ as the lower bound.

This work can be extended in many directions. Several vehicle routing problems, including the Dynamic Travelling Repairman Problem (DTRP) [5] and the Dynamic PickupDelivery Problem (DPDP) [11] for systems that are affine in control are all interesting paths to pursue.

\section{REFERENCES}

[1] K. Savla and E. Frazzoli and F. Bullo, " Traveling Salesperson Problems for the Dubins vehicle." IEEE Trans. on Automatic Control, To appear (2008).

[2] K. Savla and F. Bullo and E. Frazzoli, "Traveling Salesperson Problems for a double integrator." IEEE Trans. on Automatic Control, To Appear (2007).

[3] L. E. Dubins. "On Curves of minimal length with a constraint on average curvature and with prescribed initial and terminal positions and tangents." American Journal of Mathematics, vol. 79, pp. 497516,1957 .

[4] Erzberger, H., and Lee, H.Q." Optimum Horizontal Guidance Techniques for Aircraft." Journal of Aircraft, Vol. 8 (No. 2), pp. 95-101, February, 1971.

[5] D.J. Bertsimas and G.J. Van Ryzin. "A stochastic and dynamic vehicle routing problem in the Euclidean plane." Operations Research, vol. 39, pp. 601-615, 1991

[6] Bui X. et. Al, "Shortest Path Synthesis for Dubins Non-holonomic Robot," IEEE 1994.

[7] J. J. Enright and E. Frazzoli, " The Stochastic Traveling Salesman Problem for the Reeds-Shepp Car and the Differential Drive Robot." In Proc. IEEE Conf. on Decision and Control, December 2006.

[8] S. Sastry, " Nonlinear Systems: Analysis, Stability, and Control." Springer-Verlag New York (1999).

[9] A Isidori, " Nonlinear Control Systems." SpringerVerlag Berlin (1989).

[10] S Itani and Munther A Dahleh, "On the Stochastic TSP for the Dubins vehicle." In Proc. American Control Conference, 2007.

[11] Holly A. Waisanen et. Al, "A Dynamic Pickup and Delivery Problem in Mobile Networks under Information Constraints." IEEE Trans. on Automatic Control To Appear 2007.

\section{APPENDIX}

\subsection{Proof of lemma 2}

Here, we will prove lemma 2 for the output component $y_{s}, s \in\{1,2\}$ in the case where the system is small-time locally controllable (definition 2). This implies that $g_{0}=0$. We assume that the change of coordinates in Section 3.1 has been applied.

We will prove that using piece-wise constant inputs, $\exists T>$ 0 such that system (1) can be steered to a distance that is $C_{L_{s}} t^{o_{s}}$ in time $t \forall t \leq T$.

Proof 5. Inputs that allow system (1) can be computed iteratively. Given $t$, choose $i_{0}^{*}, \ldots, i_{o_{s}-1}^{*}$ such that

$$
\mathcal{L}_{g_{i_{0}}} \ldots \mathcal{L}_{g_{i_{o_{s}-1}}} h_{s}\left(x_{0}\right) \neq 0
$$

and design the inputs $u_{j}$ as follows:

(1) Each $u_{j}$ takes up to $o_{s}$ different values, on different time intervals $\left(\left[0, \frac{t}{o_{s}}\right], \ldots,\left[\frac{\left(o_{s}-1\right)}{o_{s}} t, t\right]\right)$. We denote the value $u_{j}$ takes between during the time $\left[\frac{(k-1)}{o_{s}} t, \frac{k}{o_{s}} t\right]$ by $u_{j}^{k-1}$.

(2) Set

$$
u_{i_{o_{s}-1}^{*}}^{0}=M, \text { and } u_{j}^{0}=0, j \neq i_{0}^{*},
$$

and the counter $l=1$. The rest of the values of $u_{j}^{l}$ are calculated iteratively (over $\left.l=1, \ldots, o_{s-1}\right)$ :

$$
\begin{gathered}
u_{i_{l}^{*}}^{l}=M, \\
u_{j}^{l}=-\frac{o_{s} \int_{0}^{\frac{l}{o_{s}} t} d \xi_{j} d \xi i_{l-1}^{*} \ldots d \xi_{i_{0}^{*}}}{t \int_{0}^{\frac{l}{o_{s}} t} d \xi i_{l-1}^{*} \ldots d \xi_{i_{0}^{*}}}
\end{gathered}
$$

Where the integrals are calculated as in theorem (2). Note that if both integrals are 0 , then we set the corresponding $u_{j}^{l}$ to 0 . Also, if

$$
\frac{o_{s} \int_{0}^{\frac{l}{o_{s}} t} d \xi_{j} d \xi i_{l-1}^{*} \ldots d \xi_{i_{0}^{*}}}{t \int_{0}^{\frac{l}{o_{s}} t} d \xi i_{l-1}^{*} \ldots d \xi_{i_{0}^{*}}}>M
$$

all of the $u_{j}^{l}$ can be normalized (for all $j$ and $l$ ).

The way the $u_{i}$ 's were designed guarantees that

$$
\int_{0}^{\frac{k+1}{o_{s}} t} d \xi_{j_{k}} \ldots d \xi_{j_{0}}=0 \forall j_{k}, \ldots, j_{0} \neq i_{k}^{*}, \ldots, i_{0}^{*} .
$$

This can be easily proved by induction over $k$ and using the design from (7).

Now using the fact that

$$
\int_{0}^{t} d \xi_{j_{o_{s}-1}} \ldots d \xi_{j_{0}}=0 \forall j_{o_{s}-1}, \ldots, j_{0} \neq i_{o_{s}-1}^{*}, \ldots, i_{0}^{*}
$$

in (3) gives:

$$
\begin{array}{r}
y_{j}(t)=h_{j}\left(x_{0}\right)+\mathcal{L}_{g_{i_{0}}^{*}} \ldots \mathcal{L}_{g_{i_{o_{s}}^{*}-1}^{*}} h_{j}\left(x_{0}\right) \int_{0}^{t} d \xi_{i_{o_{s}-1}^{*}} \ldots d \xi_{i_{0}} \\
+\sum_{k=o_{s}+1}^{\infty} \sum_{i_{0}, \ldots, i_{k}=0}^{m} \mathcal{L}_{g_{i_{0}}} \ldots \mathcal{L}_{g_{i_{k}}} h_{j}\left(x_{0}\right) \int_{0}^{t} d \xi_{i_{k}} \ldots d \xi_{i_{0}}
\end{array}
$$


From which the result directly follows.

The proof for the case of the locally non-controllable system is close but more complicated, mainly due to the fact that the system can't be steered arbitrarily.

Note that the motion in the two dimensional space $\left(y_{1}, y_{2}\right)$ can be created from motions in one direction. Therefore the two dimensional result is also easily attainable from this one. 